\title{
Learning E-commerce Web Design Engineering: Towards Discovery of Service Innovational Opportunities
}

\author{
Chien-Sing Lee \\ Department of Computing and Information Systems \\ Sunway University, Malaysia. \\ chiensingl@sunway.edu.my
}

\begin{abstract}
Graduates are expected to be skilled across disciplines and provide holistic solutions. Hence, scaffolding is crucial as transfer of learning does not occur naturally and teachinglearning strategies found to be effective for experts may not be adopted similarly for novice learners. We hypothesize that if we can help students to model in relation to the ecosystem, they should be able to identify or discover opportunities, strategically assess technologies and create new innovations and interactions for e-commerce Websites. For goal-based scaffolding, we use technology-assisted design thinking, embedded formative assessments. We study more effective ways to develop: a) skills useful to the design and development of sustainable Web systems and services and possibly sustainable smart services in the future by relating business to technology and impacts on society; b) epistemic agency for lifelong learning.
\end{abstract}

Keywords- Strategic Technology Assessment; Web modeling and design; discovery of innovational opportunities, sustainable services.

\section{INTRODUCTION}

Increasing competitiveness in science and technology development in several countries, most notably in terms of smart cities and robotics, have necessitated new approaches to product and service design. For example, in the IEEE Smart Cities (SC) initiative [1], the aim is to create more sustainable futures through technological innovations. Similarly, [2] aims to increase quality of life. They envision it as peopledriven/centric emphasizing on green living, efficiency, ubiquity and sustainability.

Consequently, to equip students to be able to design such technological innovations, instructors need to equip students for transformative learning. The question is how? We look at diverse disciplines.

Gaynor [3], Vice-President of the IEEE Technology, Engineering and Management Society (TEMS), highlights skills expected of graduates: Strategic Technology Analysis and Management processes, Innovation, Entrepreneurship, Project Management, Organizational Interfaces and Excellence in Execution.

Furthermore, for creative solutions, [4] from the learning sciences, characterizes creativity as involving nonmechanistic analogical substitution, incremental knowledge and skill refinement processes and goal-directed reflection, backtracking and refinements to increase consideration of alternatives and consequently elaboration and evaluation of these alternatives in relation to the learning or task goal.

\author{
K. Daniel Wong \\ Daniel Wireless Software Pte Ltd., \\ Singapore. \\ dwong@danielwireless.com
}

From an educational perspective, [5] regard learning as experiential, via learning-by-doing, driven by goals. Furthermore, in [6]'s review of literature, they find that formative assessments (progressive assessment during the course), is one of the most effective means to obtain information about students' knowledge and the depth of their understanding of subject matter. In essence, it enables students' activities to generate evidence that can be synthesized back into formative feedback.

\section{A. Objectives}

This study is in the e-commerce domain. Dimensions of ecommerce are wide-ranging, from transaction processing systems, supply chain management systems, enterprise resource planning systems, customer relationship management systems, knowledge management systems to business intelligent systems [7, 8]. Moreover, e-commerce is interdisciplinary. Human-computer interaction for example, draws from cognitive psychology/customer behavior research, software engineering, database engineering and analytics.

Returning to the question of how to scaffold transformative learning in developing the skills highlighted by [3], especially innovation opportunities, we find that:

a) not much research have focused on the role of formative assessment in shaping goal-based metacognition;

b) research work by the learning sciences communities aimed at developing creativity, such as by [4], is scaffolded by guiding questions. Hence, we should focus on the design of guiding questions.

In this exploratory study, we aim to scaffold strategic technology assessment (which precedes design) and the discovery of opportunities, such as those identified by [9] in Table 1. Although m-commerce has increasingly become more pervasive and disruptive, the scope for this study is only on Web-based e-commerce.

\section{B. Research question}

In our prior research in the creative industries and in Software design and testing, we find that fluid compositions and patterns respectively, are useful in helping students to theorize and model. Prototyping was via Web design and/or social media, hand-crafting (depending on discipline) [10], and Web design [11] respectively. The modeling outcomes differ in degrees of breadth and depth. 
In this exploratory study, our research question is how will e-commerce Web design engineering scaffolded by design-thinking goal-based reflection and embedded formative assessments influence the learning of strategic technology assessments and innovational outcomes?

Web design engineering requires systematic, disciplined and quantifiable approaches towards successful development of high-quality, ubiquitously usable Web-based systems and applications. Hence, it requires design. More importantly, sustainable design creates long-term value. As such, ecommerce Web design engineering provides sufficient avenue for students to hypothesize, experiment with and learn $[3,4]$ 's skills, and perhaps more sustainable services.

TABLE 1. Aspects of E-commerce and the Innovational Opportunities They Engender (the 5Cs Framework).

\begin{tabular}{|l|l|}
\hline Activity & $\begin{array}{l}\text { E-commerce aspect of domain Web-Internet } \\
\text { compound }\end{array}$ \\
\hline Commerce & Marketplace \\
\cline { 2 - 2 } & Providing a universal value-chain linkage \\
\hline \multirow{3}{*}{ Collaboration } & Network of relationships \\
\cline { 2 - 2 } & Collaboratory \\
\hline Connection & Forum \\
\cline { 2 - 2 } & Interactive medium \\
\cline { 2 - 2 } & Delivery vehicle \\
\cline { 2 - 2 } & Anytime-anywhere connectivity \\
\cline { 2 - 2 } & Development platform \\
\cline { 2 - 2 } Computation & Computing utility \\
\hline
\end{tabular}

\section{Significance}

First, [3, 4]'s skills are useful to the design and development of systems and services in an increasingly digitalized world; applicable/extendable to MOOCs and adaptive yet self-regulatory student modelling (e.g. by [12]).

Second, students learn how to relate business to technology and society through design. By focusing on discovery of opportunities crucial to strategic technology assessment and innovation, we hope to in the future, provide a natural motivation to explore smart services (co)-design and reification to improve quality of life.

\section{Scope}

We scope our study to scaffolding transfer of learning with regards to learning and designing for e-commerce through Web design engineering. We are concerned with the front end, i.e., the user interface and interaction designs, where the learning of skills highlighted by [3] can fully be explored and improved.

In the next section, we review related work on the design and scaffolding.

\section{RELATED WORK}

$[13,14]$ characterize the Design Cycle as grounded on a reflective and repetitive structure of design processes; facilitated by visual and/or verbal means of communication (via design tools), and a new design cycle after critical rethinking of the perceived idea. They also highlight that in contrast to the rationalist approach, there is a sense-making reflective aspect to modelling the world. For example, [14] views designers as often switching between "framing," "making moves," and "evaluating moves." During coevolution, the learner refines a mental picture of the design object based on a conceptualization of the context, and vice versa" by prototyping a design object.

Changing trends in e-commerce Web design focuses more on user experience than transaction processing [9] or process/workflow modelling and management. Examples of such Web sites are Lazada and Amazon. Table 1 shows that e-commerce has extended to collaboration and communication; necessitating disruptive interactive technologies through social media and m-commerce. Hence, conceptualization has become crucial.

To meet the need to innovate, [15]'s study supports the importance of experimental prototyping and establishing a point of view, where framing and reflective evaluations take place. Aiming to synergize the designers and managers' perspectives and tasks, they propose a design-thinking-based unified innovation process model which highlights prototyping as an experimental reflective medium for dialogic inquiry.

\section{METHODOLOGY}

We apply [16]'s entrepreneurial framework. An entrepreneurial innovation narrative views opportunities as "found" or "made". The actor evolves through a dynamic balance of micro-macro (actor-centric / context-centric) approaches to multilevel to constitutive approaches (e.g. those informed by structuration, complexity theories), with the main aim of developing generalizations/ principles.

\section{A. Sample}

The sample group is 31 undergraduate students in their first, second and third year of study in the Information systems program in Sunway University, a private university in Malaysia. E-commerce is a core subject.

\section{B. Procedure}

Consistent with [17], students engage with multiple examples through lectures, compare examples and develop generalizations. These examples serve to enhance the development of analogies which bridge the application of principles to actual use in real life.

To guide their reflection, goal-based questions revolving around interactivity, fun and sustainability/user experience are posed. A lot of emphasis is placed on their perceived definition of sustainability and how this would align their design, and product to the assessment criteria.

We next present our design in terms of context, tasks and its regulatory mechanism, assessment.

\section{Design}

Context: Students are taught the business models in [7], examples from [8] and those from the Internet. These examples are discussed. Lectures' and tutorials' thrusts 
explain systems analysis and design in terms of its two main components: logical design and physical design. Objectoriented design is introduced in a tutorial on the design of a water bottle.

Task: For assignment, students are asked to choose their own partners and seven groups are formed. The task is: You are the systems analyst and Web designer for a company in Malaysia. As such, you are responsible for Web innovation. There are several parts to the tasks, simulating [16]'s narrative journey (elaborated on in an extended paper). Each phase is guided by specific questions.

Assessment: Assessment rubrics stress on several factors, i.e., fun, interactivity, sustainability which contribute to user experience; novelty and significance which contribute to problem-solving. Students are encouraged to constantly selfevaluate their designs and prototypes based on these criteria.

Our research model is illustrated in Fig. 1.

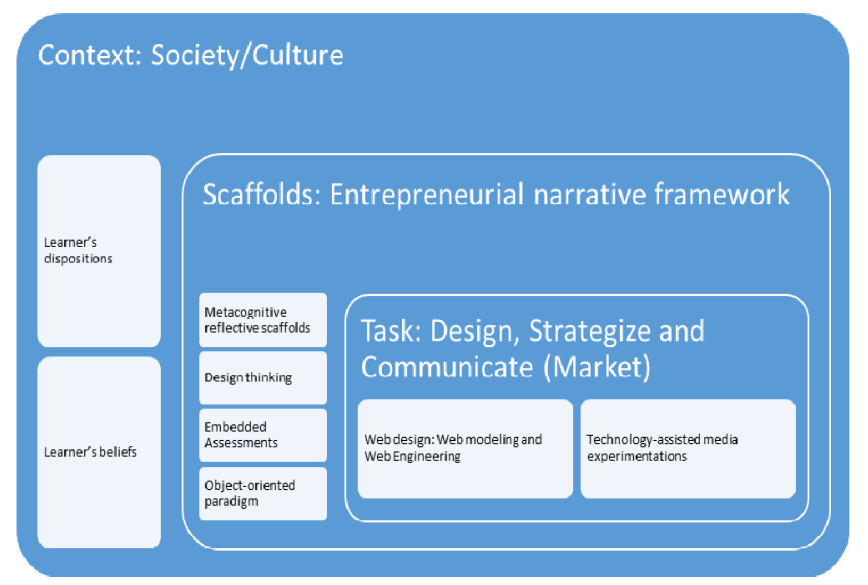

Fig. 1. Our research model

\section{FINDINGS AND CONCLUSION}

Students fully achieved the learning outcomes. Furthermore, students from all groups not only exhibited self-directed causal associations, but also synergies of models to varying degrees of success. Referring back to [10]'s table of opportunities, all groups' Web sites exhibit opportunities fulfilling commerce, collaboration and communication. The overriding thrust that business objectives drive technological design and social impact and vice versa is evident in all groups.

In addition, all groups include the supply chain management system in their design, 3/7 groups include customer relationship management, and 3/7 groups include a combination of both and out of this, 2/7 groups place heavier emphasis on intangible factors.

Findings thus indicate that Web modeling and Web designing to represent e-commerce business models and strategies can provide the experimental platform for dialogic inquiry for the discovery of opportunities and strategic technology assessment. This also supports cognitive fit theory, (that if the technology fits the task and vice versa, then information acquisition and performance will improve) and our hypotheses. An extended paper elaborates on the models students derived.

Our sample size is small. As such, findings are not generalizable yet. Future research will go deeper into product-service and interaction design and Web-mobile human-computer interaction design for the elderly and youth.

\section{REFERENCES}

[1] G. Betis, "The IEEE Smart Cities Initiative: From smart cities to smart citizens," Talk presented at the Malaysian Communications and Multimedia Commissons on Smart City Framework, September 10, 2015, Putrajaya, Malaysia.

[2] J. Wong, "Malaysia's Journey to become a smart digital nation", Talk presented at the Malaysian Communications and Multimedia Commissons on Smart City Framework, September 10, 2015, Putrajaya, Malaysia.

[3] G. H. Gaynor. In L. P. E. Maki, "Professional and Economic Success: Technology and Engineering Management," The Boeing Company, IEEE TEMS Leader, April 2015.

[4] J. L Kolodner, Understanding creativity: A case-based approach Lecture Notes in Computer Science, vol. 837, 1994, pp. 1-20.

[5] R. C.. Schank, A. Fano, B. Bell \& M. Jona, "The Design of GoalBased Scenarios," Journal of the Learning Sciences, vol. 3, issue 4, 1994.

[6] P. Black and D. William, "Inside the black box: Raising standards through classroom assessment," Kings College, London, 1998.

[7] K. C., Laudon, and C. G. Traver, "E-commerce 2015: Business, technology, society (11 ${ }^{\text {th }}$ edition)," Global Edition. Pearson, New York.

[8] E. Turban, D. King, J. Lee, T. P. Liang, T.P., and D. C. Turban, "Ecommerce: A Managerial and Social Networks Perspective," Pearson, NY, 2012.

[9] V. Zwass, "Electronic Commerce and Organizational Innovation: Aspects and Opportunities," International Journal of Electronic Commerce. Spring 2003, vol. 7, no. 3, pp. 7-37.

[10] C. S. Lee, K. D. Wong and S. B. Y. Lau, "Scaffolds and design factors to increase creative outcomes in teaching software design and testing," International Conference on Industrial Engineering and Engineering Management," December 6-9, 2015, Singapore.

[11] Lee, C. S. and K. D. Wong. (2015). Developing a Disposition for Social Innovations: An Affective-Socio-Cognitive Co-Design Model. International Conference on Cognition and Exploratory Learning in Digital Age, 24 - 26 October 2015 in Maynooth, Greater Dublin, Ireland.

[12] S. Graf, and Kinshuk, "Dynamic Student Modelling of Learning Styles for Advanced Adaptivity in Learning Management Systems," International Journal of Information Systems and Social Change, vol. 4, no. 1, January-March 2013, pp. 85-100.

[13] B. Blanchard and W. Fabrycky, "Systems Engineering and Analyses," (4th Edition), Prentice-Hall, NJ, 2005.

[14] P. Ralph, "The two paradigms of software design.," Lero Invited Talks, University College Cork, 28 May 2015.

[15] P. Skogstad and L. Leifer, "A unified innovation process model for Engineering designers and managers," In Christoph, M. and Leifer, L. J. Eds. Design thinking: understand, improve, apply. Understanding innovation. Springer-Verlag. Berlin, Heidelberg, 2011.

[16] R. Garud, J. Gehman, J. and A. P. Giuliani, "Contextualizing entrepreneurial innovation: a narrative perspective," IEEE Engineering Management Review, vol. 3, 1, 2015, pp. 80-102.

D. N. Perkins and G. Salomon. In International Encyclopedia of Adult Education and Training. (Ed.,Tuijnman, A. C, Pergamon Press, Tarrytown: NY., 1996, pp. 422-427. 